\title{
Insulin-like signaling pathway functions in integrative response to an olfactory and a gustatory stimuli in Caenorhabditis elegans
}

\author{
Ya-Ming Jiu ${ }^{1^{*}}$, Yang Yue ${ }^{1^{*}}$, Song Yang ${ }^{1^{*}}$, Lin Liu ${ }^{1}$, Jun-Wei Yu ${ }^{1}$, Zheng-Xing $\mathrm{Wu}^{1}(\bowtie)$, Tao Xu ${ }^{1,2}(\bowtie)$ \\ ${ }^{1}$ Key Laboratory of Molecular Biophysics, Ministry of Education, and Institute of Biophysics and \\ Biochemistry, Huazhong University of Science and Technology, Wuhan 430074, China \\ 2 National Laboratory of Biomacromolecules, Institute of Biophysics, Chinese Academy of Sciences, \\ Beijing 100101, China \\ $\triangle$ Correspondence: xutao@ibp.ac.cn (T. Xu), ibbwuzx@mail.hust.edu.cn (Z.-X. Wu) \\ Received September 23, 2009; accepted October 28, 2009
}

\begin{abstract}
Animals integrate various environmental stimuli within the nervous system to generate proper behavioral responses. However, the underlying neural circuits and molecular mechanisms are largely unknown. The insulinlike signaling pathway is known to regulate dauer formation, fat metabolism, and longevity in Caenorhabditis elegans (C. elegans). Here, we show that this highly conserved signaling pathway also functions in the integrative response to an olfactory diacetyl and a gustatory $\mathrm{Cu}^{2+}$ stimuli. Worms of wild-type N2 Bristol displayed a strong avoidance to the $\mathrm{Cu}^{2+}$ barrier in the migration pathway to the attractive diacetyl. Mutants of daf-2 (insulin receptor), daf-18 (PTEN lipid phosphatase), pdk-1 (phosphoinositide-dependent kinase), akt-1/-2 (Akt/PKB kinase) and sgk-1 (serum- and glucocorticoidinducible kinase) show severe defects in the elusion from the $\mathrm{Cu}^{2+}$. Mutations in DAF-16, a forkhead-type transcriptional factor, suppress the integrative defects of daf-2 and akt-1/-2 mutants. We further report that neither cGMP nor TGF $\beta$ pathways, two other dauer formation regulators, likely plays a role in the integrative learning. These results suggest that the insulin-like signaling pathway constitutes an essential component for sensory integration and decision-making behavior plasticity.
\end{abstract}

KEYWORDS C. elegans, insulin signaling pathway, sensory integration, decision making behavior

\section{INTRODUCTION}

Learning and memory is a fundamental process by which animals adapt to the changing environment, evaluate various sensory signals based on context and experience, and make decisions to generate adaptive behavior. Extensive crossmodal interaction during sensory processing has been found in invertebrates, vertebrates and primates (Calvert et al., 2001; Guo and Guo, 2005; Celikel and Sakmann, 2007). The understanding of mechanisms on the level of molecules, neurons and neuronal circuits is the basis for recognition of learning and memory, or decision making.

Caenorhabditis elegans ( $C$. elegans) is an ideal model to conduct genetic, cellular and molecular analyses of behavior. Its small body size, short life cycle, hermaphroditic reproduction, and accessibility to classical genetics all make it possible to efficiently analyze mutants exhibiting abnormal behaviors. C. elegans has a simple nervous system composed of 302 neurons in the adult hermaphrodite, the anatomical wirings of which have been mapped by electron microscopy (Ward et al., 1975; White et al., 1986). The worms are able to process and integrate sensory inputs from different modalities and generate adaptive behaviors. The $C$. elegan shen-1 gene, which encodes a secretory protein with a low-density

${ }^{\star}$ These authors contributed equally to the work 
lipoprotein (LDL) receptor motif, has been reported as an essential component for sensory integration of odorant and taste stimuli (Ishihara et al., 2002). It has demonstrated that sensory signaling and sensory plasticity in $C$. elegans are very similar to those in vertebrates. Most of the signaling pathways indentified through worm genetics are conserved in evolution. Therefore, the genetic analysis in C. elegans may provide important insights into the molecular mechanisms of the behaviors, such as sensory signaling integration, learning, memory, etc.

Involvement of insulin receptor and its downstreamtargeted molecules in neural and behavioral plasticity has been highlighted by various investigations in mammals. The insulin receptor is highly expressed in the hippocampus and cerebellum, and its inhibition in the central nervous system causes deficits in learning and memory (Zhao et al., 2004). It has been reported that this highly conserved insulin-like signaling pathway is involved in food-temperature association behavior, which is modulated by the memory of previous culture temperatures (Kodama et al., 2006). The Insulin/PI 3Kinase pathway also regulates salt chemotaxis learning in $C$. elegans (Tomioka et al., 2006). These studies represent important progress toward understanding the molecular mechanisms of the insulin-like signaling pathway in learning and sensory processing. In spite of these studies, the role of this pathway in the integration of different signalings remains unclear.

In the present work, we assayed the integrative behavior of diacetyl and $\mathrm{Cu}^{2+}$ in mutants of the insulin signaling pathway such as daf-2, daf-18, pdk-1, akt-1/-2, sgk-1 and several double mutants. Our results demonstrate that the insulin-like signaling pathway plays an important role in the behavioral integration of epigamic olfactory and nocuous gustatory signals in C. elegans.

\section{RESULTS}

Components of the insulin-like signaling pathway are required for sensory integration learning in $C$. elegans

Several learning paradigms in $C$. elegans, such as salt chemotaxis learning (Saeki et al., 2001; Tomioka et al., 2006), olfactory adaptation (Colbert and Bargmann, 1995), and temperature learning (Mohri et al., 2005) have been utilized to investigate associative learning and memory. The components of the insulin-like signaling pathway in C. elegans are highly conserved with those in mammals, the mammalian orthologs in C. elegans including DAF-2, a homolog of the insulin receptor; AGE-1, a PI 3-kinase homolog; DAF-18, a PTEN (phosphatase and tensin homolog deleted on chromosome 10) lipid phosphatase homolog; PDK-1, AKT-1/2 and SGK-1, homologs of phosphoinositide-dependent protein kinases; and DAF-16, a FOXO family transcription factor (Fig. 1B). Early findings indicate that these insulin-like signaling pathway genes are essential for associative learning and memory in C. elegans (Kodama et al., 2006; Tomioka et al., 2006). Here, for evaluating the possible function of this signaling pathway in the sensory integration, we used the interaction assay developed by Ishihara et al. (Ishihara et al., 2002) to assess the behavioral integration of two sensory signals. C. elegans normally shows chemotaxis toward odorants like diacetyl; however, chemotaxis toward diacetyl was suppressed in the presence of $\mathrm{a} \mathrm{Cu}^{2+}$ barrier (Fig. 1A) (Ishihara et al., 2002). Insulin-like signaling mutants showed severe defects in crossing the $\mathrm{Cu}^{2+}$ barrier when migrated towards diacetyl. As shown in Fig. 1C, at $100 \mathrm{mM}$ $\mathrm{Cu}^{2+}$ and $10^{-2}$ diacetyl, daf-2, akt-1/-2 and sgk-1 loss-offunction worms migrated to the diacetyl side in much higher proportions. In contrast, daf-18, pdk-1(gf) and daf-16 mutant worms were reluctant to cross the $\mathrm{Cu}^{2+}$ barrier. The daf-16 mutant strains were defective in the interaction assay to different extents (Fig. 1C). daf-16(m27), bearing a point mutation, behaved the same way as wild type animals in the integration test. The other two alleles of daf-16 (mu86 and $m g D f 50$ ), which are reported to be loss-of-function mutants, showed a weak tendency to cross the $\mathrm{Cu}^{2+}$ barrier. Furthermore, daf-16 mutants showed a dauer-formationdefective phenotype, in contrast to daf-2, pdk-1, akt-1/-2 and $s g k-1$ mutants. These results are consistent with the negative role of daf-16 and daf-18 in insulin signaling and support the conclusion that the insulin-like signaling pathway is involved in the sensory integration learning process in C. elegans.

\section{Insulin-like signaling pathway mutants have normal sensation and locomotion}

There are several possible causes for the defects in behavioral integration of two sensory signals, i.e., defects in sensation, in integration of sensory signals, in both sensory processes, and/or in locomotion. To distinguish between these possibilities, we first analyzed the ability of the worms to sense the two stimuli. Our data demonstrated that all the mutants tested had no defects in sensation of diacetyl. As shown in Fig. 2A, no differences were observed between wildtype N2 Bristol (as controls) and mutant worms (daf-2, daf-18, akt-1/-2 and sgk-1) for chemotaxis toward diacetyl. Avoidance of $\mathrm{Cu}^{2+}$ in the absence of diacetyl was also analyzed. We did not observed statistically significant differences for obviation of $\mathrm{Cu}^{2+}$ in any of the mutants except age-1(hx546) and akt-1 ( $m g 144$ ) when compared with controls (Fig. 2B).

Second, we recorded and analyzed the body bends of the mutants daf-2, daf-18, akt-1/-2 and sgk-1 to exclude the possible influence of locomotion defects on the integration assay. As shown in Fig. 3A, all mutants showed no significant differences in body bends. This observation was further supported by the neuroactive drug assay. The daf-2, akt-1 and daf-16 mutants showed a similar response to the acetylcholinesterase inhibitor aldicarb as the wild type (Fig. 3B). These 
A

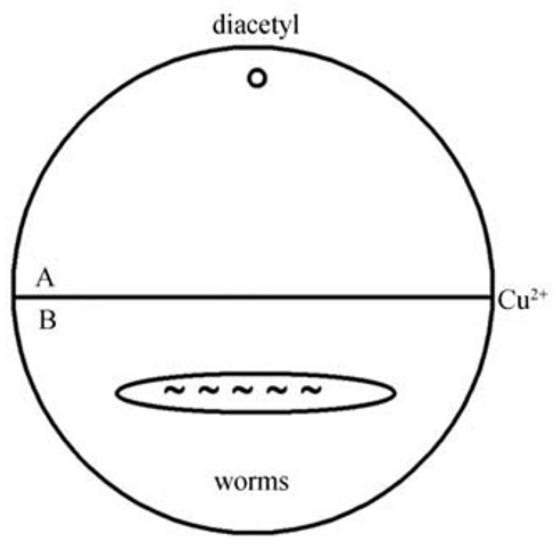

B

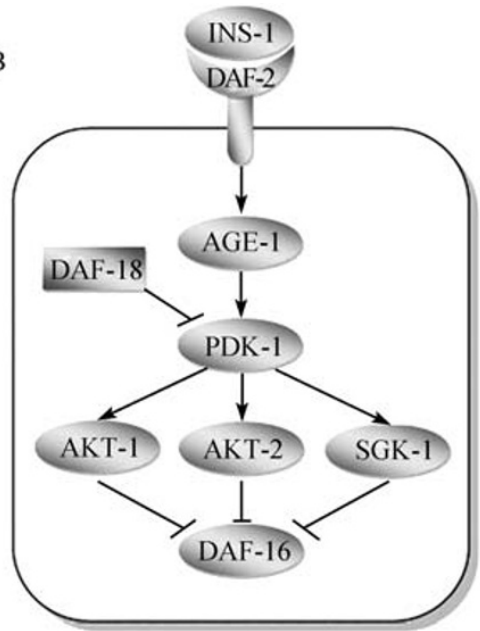

C

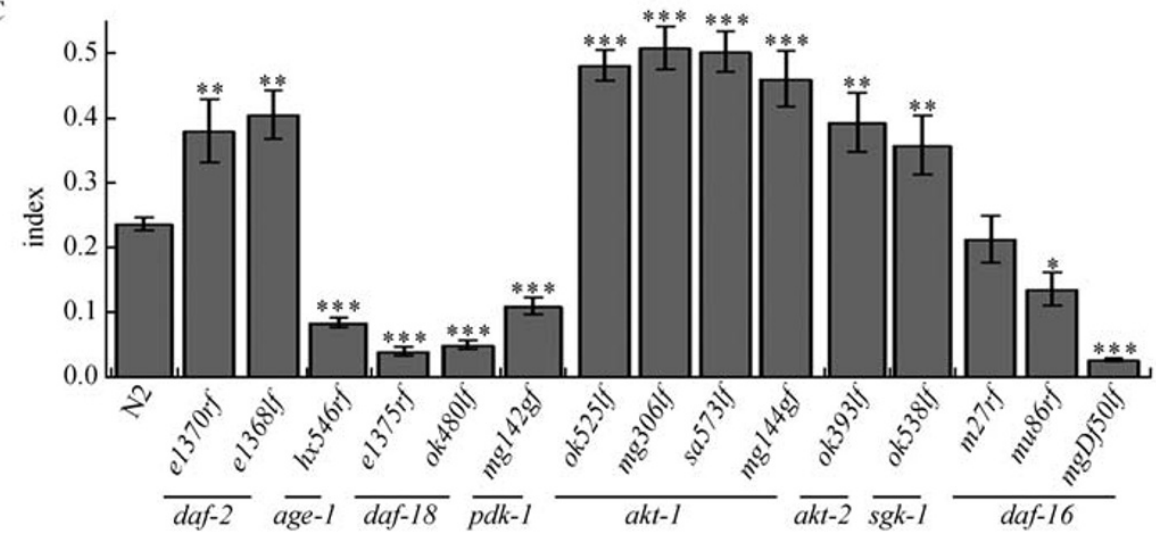

Figure 1. The integration of diacetyl and $\mathrm{Cu}^{2+}$ signaling was impaired in insulin-like signaling pathway mutants. (A) Schematic diagram for the integration assay system. (B) Model of the insulin-like signaling pathway. (C) The choice index of worms crossed $\mathrm{Cu}^{2+}$ and reached diacetyl spot. If indicates reduction of function mutants, If, loss of function mutants, and $g f$, gain of function mutants. Error bars indicate the SEM. Asterisks indicate the significance of differences from the wild type N2. ${ }^{* *}$, $p \leqslant 0.001 ;{ }^{* *}, p \leqslant 0.01 ;{ }^{*}, p \leqslant 0.05$.

results thus excluded the possibility that the defects in the integration of diacetyl and $\mathrm{Cu}^{2+}$ signaling of insulin signaling mutants was caused by abnormalities in their sensation or locomotion.

\section{daf-16 suppresses information integration defects of mutants of upstream components in the insulin-like pathway}

DAF-16 is a downstream target negatively regulated by the DAF-2 pathway, and daf-16 loss-of-function mutations suppress the dauer-constitutive formation and long-life phenotypes (Paradis and Ruvkun, 1998). To determine whether the insulin-like signaling pathway plays its role in the integrative function as a whole, or just the components functions individually, we analyzed the double mutants daf-2;daf-16, akt-1;daf-16 and akt-2;daf-16 that were obtained by crossing the daf-16(mu86) mutant (which had been backcrossed 11 times) with the daf-2, akt-1, and akt-2 mutants respectively. As shown in Fig. 4, the daf-16 mutant indeed suppressed insulin pathway mutants in the interaction assay. In addition, a daf-2;pdk-1 (gf) double-mutant suppressed the daf-2 integrative defect, confirming that gain-of-function pdk-1 could also suppress daf-2 (Fig. 4). Taken together, these results indicate that daf-16 acts downstream of the insulin-like signaling pathway to negatively regulate sensory integration in $C$. elegans and the signaling pathway functions in the sensory integration as a whole.

cGMP and TGF $\beta$ pathways show normal integration in diacetyl and $\mathrm{Cu}^{2+}$ signaling in $\mathrm{C}$. elegans

In addition to insulin-like signaling, the cyclic guanosine monophosphate (cGMP) and transforming growth factor beta 

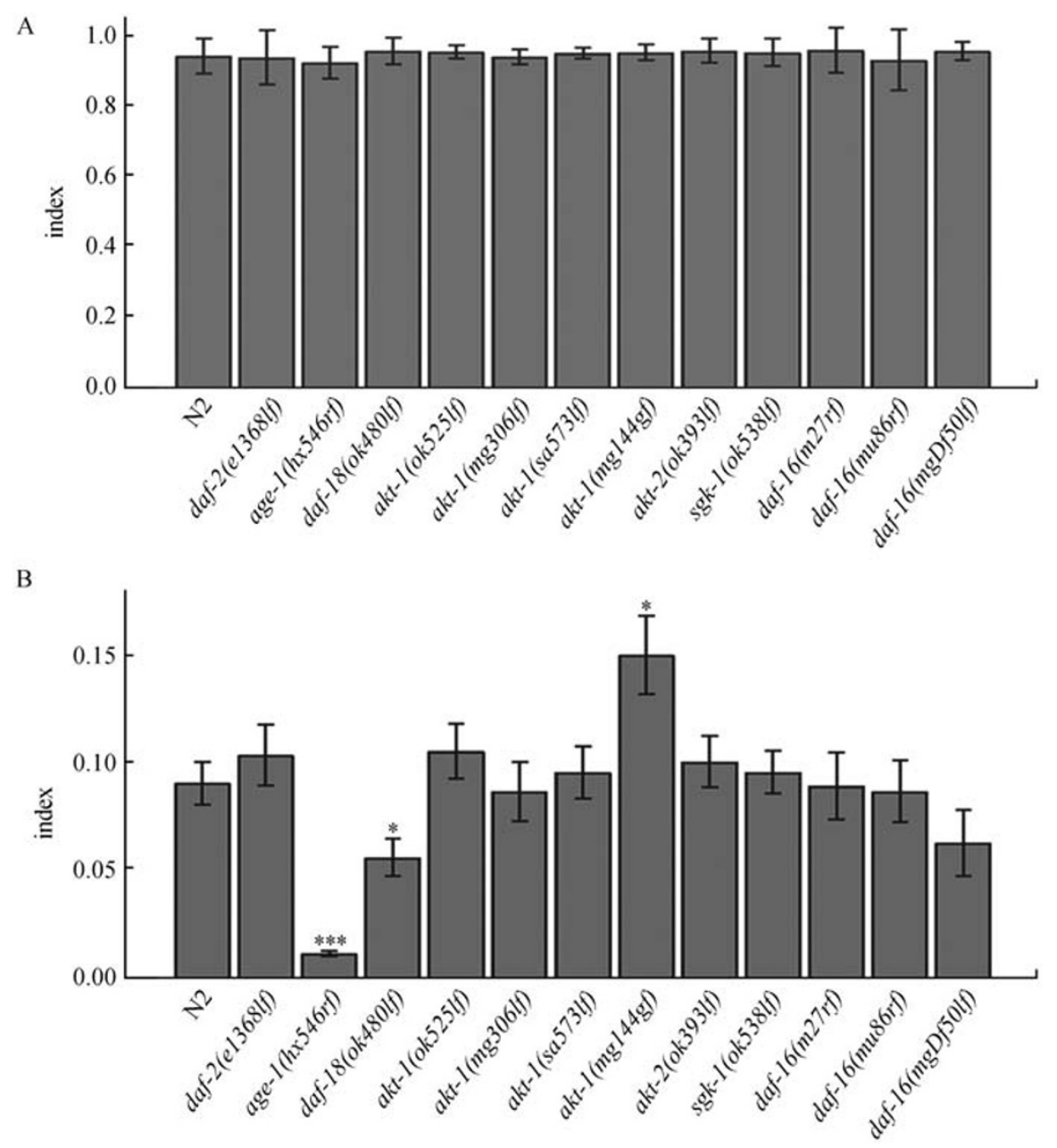

Figure 2. Sensation assay of insulin-like signaling pathway mutants. (A) All mutants showed no difference in chemotaxis to diacetyl. (B) All mutants except age-1(hx546rf), daf-18(ok480lf) and akt-1(mg144gf) displayed similar elusion of $\mathrm{Cu}^{2+}$. Error bars indicate the SEM. Asterisks indicate significant differences from the wild type N2. ${ }^{* \star}, p \leqslant 0.001 ;{ }^{*}, p \leqslant 0.05$.

(TGF $\beta$ ) pathways are also implicated in regulating dauer formation in $C$. elegans. To address whether these two pathways are involved in the integration of the diacetyl and $\mathrm{Cu}^{2+}$ signaling pathways, we quantified the proportions of daf11 , daf- 7 and daf-3 mutants that crossed the $\mathrm{Cu}^{2+}$ barrier and reached the diacetyl spots. daf-11, daf-7 and daf-3 encode a receptor guanylyl cyclase, a member of the TGF $\beta$ super family and a co-SMAD protein respectively. As shown in Fig. 5, all of these mutants displayed normal interaction behavior, suggesting that CGMP and TGF $\beta$ signaling pathways are not required in the integration of diacetyl and $\mathrm{Cu}^{2+}$ signaling in $C$. elegans.

\section{DISCUSSION}

To properly adapt to the environment, it is essential for animals to sense many environmental stimuli simultaneously. These sensory signals need to be integrated at some point in the nervous system, and decisions need to be made to generate proper behavioral responses. In $C$. elegans, the genes and neural circuits for sensory transduction have been studied extensively by behavioral and genetic analysis of responses to single stimuli (Bargmann et al., 1993; Bargmann and Kaplan, 1998). However, as in other animal systems, sensory integration in the neural circuit remains largely unknown.

Several genes are known to be involved in sensory integration-based learning. Mutations in $\mathrm{HEN}-1$, a secretory protein with an LDL motif, and CASY-1, the $C$. elegans ortholog of calsyntenins/alcadeins, lead to defects in the integration of two sensory signals (Ishihara et al., 2002; Ikeda et al., 2008). Similar defects in the integration of olfactory diacetyl and gustatory $\mathrm{Cu}^{2+}$ signaling were observed in our study of the insulin-daf-2 signaling pathway mutants. In addition, the insulin-signaling pathway also regulates salt chemotaxis learning (Ishihara et al., 2002; Matsuki et al., 2006; Tomioka 


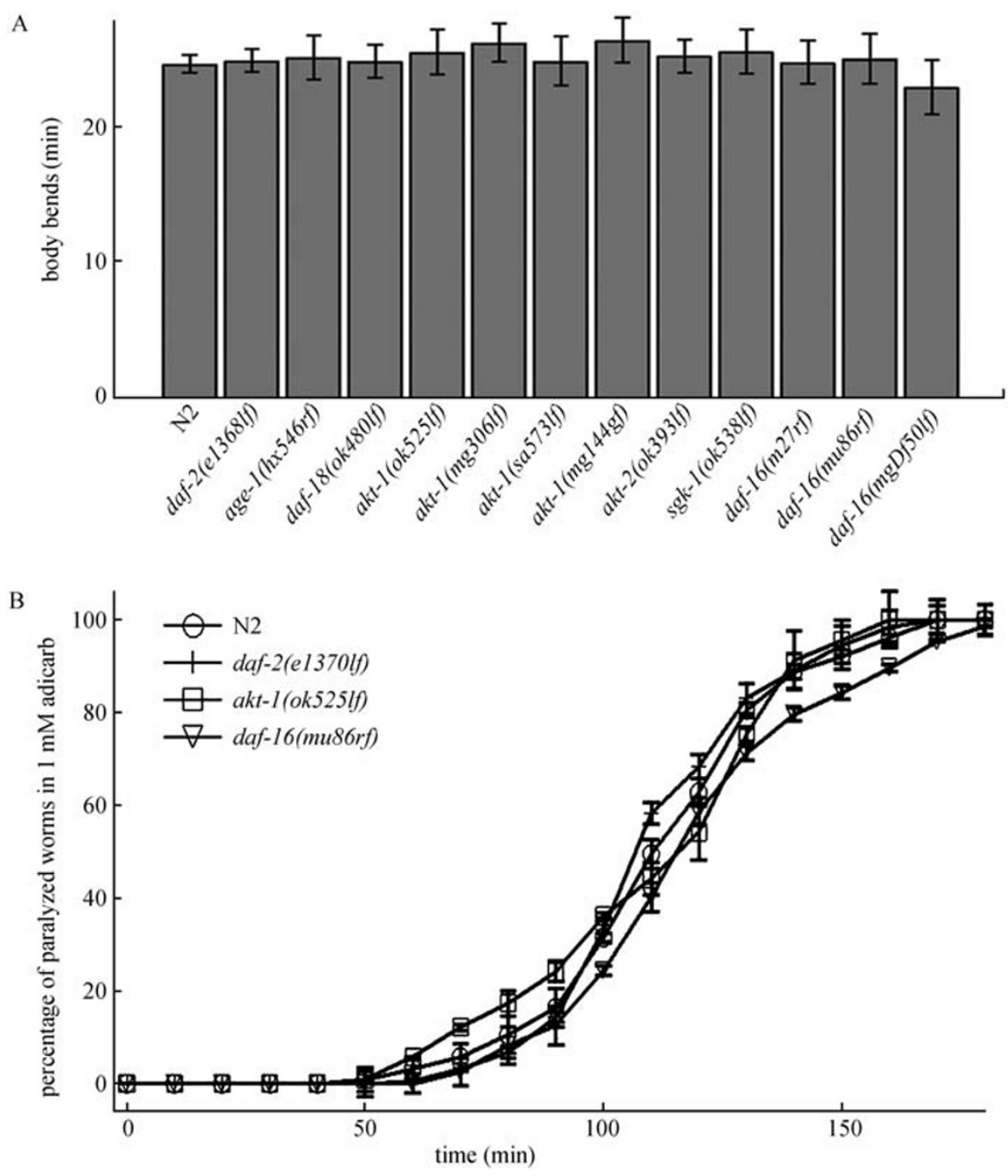

Figure 3. Assay of body bends and aldicarb resistance. (A) Body bends per minute in N2 worms as controls $(n=29)$ and the insulin-like signaling pathway mutants $(n=15,23,26,18,14,19,26,17,25,21,24$ and 28 for mutants listed from left to right, respectively). (B) Resistance to aldicarb (1 $\mathrm{mM}$; Chem Services) is normal in insulin-like signaling pathway mutants. Error bars indicate the SEM.

et al., 2006) and thermotaxis (Ishihara et al., 2002; Kodama et al., 2006). These observations suggest that different forms of learning and memory in C. elegans depend on overlapping molecular mechanisms and it is possible that insulin signaling constitutes an essential molecular pathway in many forms of learning.

To our knowledge, we are the first to report evidence that the insulin signaling pathway as a whole is also involved in sensory integration. As shown in Fig. 1C, all loss-of-function mutants of the positive regulators of the insulin pathway display higher proportions of (daf-2, akt-1/2 and sgk-1) crossing the $\mathrm{Cu}^{2+}$ barrier, in contrast to the lower proportions in the daf-16 and daf-18 (negative regulators), and pdk-1 gain-of-function mutants. One exception was age-1(hx546) (Solomon et al., 2004), which was reluctant to cross the barrier. This seemingly contradicts the positive role of age- 1 in insulin signaling; however, as we validated further (Fig. 2B), the age-1(hx546) mutant exhibits hypersensitivity to $\mathrm{Cu}^{2+}$, and thus its aversion behavior might simply be attributed to its abnormal sensitivity to $\mathrm{Cu}^{2+}$.

It is intriguing that the gain-of-function mutant akt-1(mg144), which has an Ala-183-Thr substitution (Paradis and Ruvkun, 1998), showed a similar stronger tendency to cross the $\mathrm{Cu}^{2+}$ barrier as its loss-of-function mutant equivalents. As shown in Fig. 2B, the akt-1 (mg144) worms show a slight insensitivity to $\mathrm{Cu}^{2+}$ ion, which may explain the stronger tendency of these worms to cross the $\mathrm{Cu}^{2+}$ barrier. Besides, akt-1(mg144) has been shown to suppress the dauer formation impairment of the age-1 mutant but not its increased life span (Paradis and Ruvkun, 1998). It has been suggested that akt-1(mg144) bypasses the need for AGE-1 signaling in reproductive development but does not activate normal aging pathways 


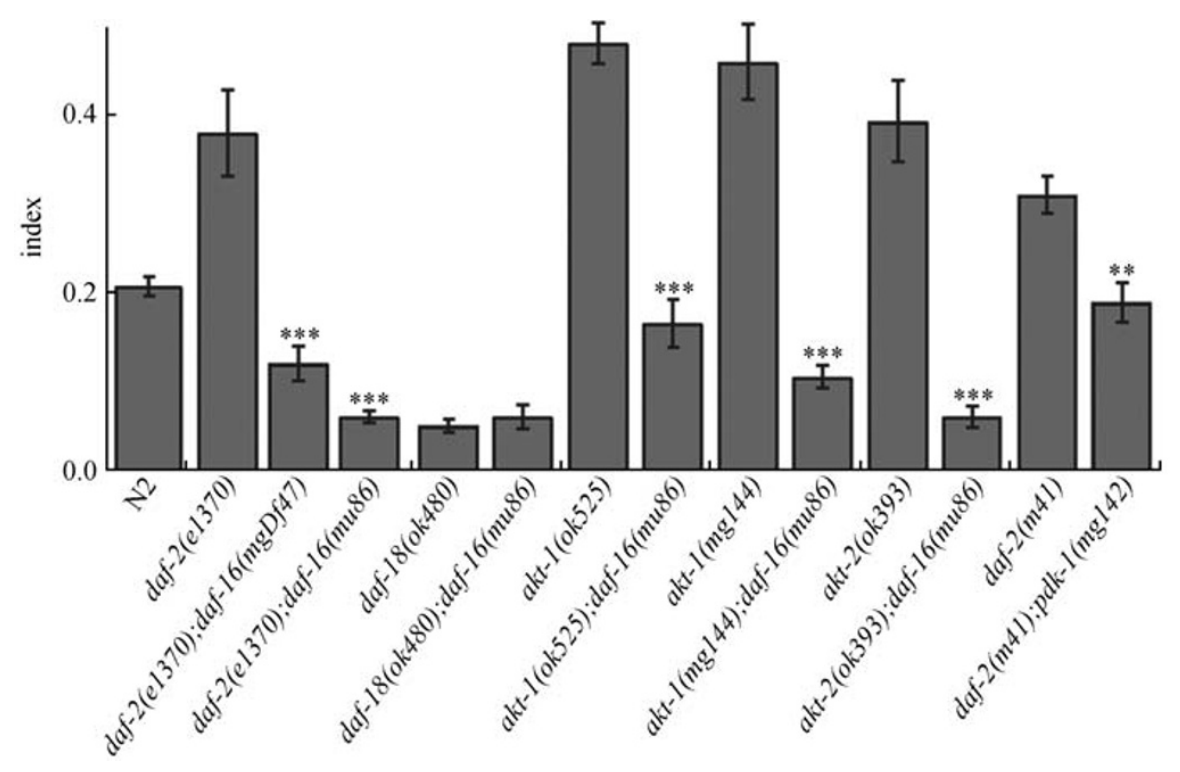

Figure 4. The integration assay of insulin-like signaling pathway double mutants. Error bars indicate the SEM. Asterisks indicate significant differences in comparisons with single mutant worms. ${ }^{* *}, p \leqslant 0.001 ;{ }^{* *}, p \leqslant 0.01$.

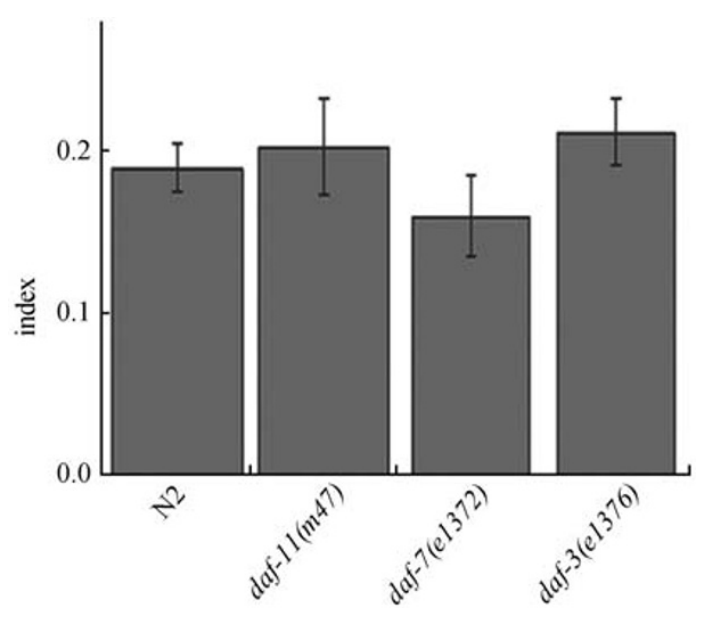

Figure 5. Mutants of the cGMP and TGF $\beta$ pathways (daf11, daf-7 and daf-3) displayed no defects in integration of diacetyl and $\mathrm{Cu}^{2+}$ signaling. Error bars indicate the SEM.

(Paradis and Ruvkun, 1998). It is thus possible that akt-1 (mg144) does not contribute to all the functions of the wild type AKT-1, especially in sensory integration. The exact mechanisms underlying the action of different akt mutations in different physiological processes remain to be explored.

\section{MATERIALS AND METHODS}

\section{C. elegans strains}

All strains were maintained by standard methods (Brenner, 1974). The following strains were obtained from the $C$. elegans Genetic Center
(CGC): N2 (wild-type Bristol, as control), daf-2(e1370)X, daf-2(e1368) $\mathrm{X} 3$, daf-2(m41)X3, age-1(hx546)X3, daf-18(e1375)X, daf-18(ok480) $\mathrm{X0}, \mathrm{pdk}$-1(mg142)X4, akt-1(ok525)X0, akt-1(mg306)X6, akt-1(sa573) $\mathrm{X} 2$, akt-1(mg144)X3, akt-2(ok393)X0, sgk-1(ok538)X0, daf-11(m47) $\mathrm{X}$, daf-7(e1372)X, daf-3(e1376)X, daf-16(m27)X, daf-16(mu86)X11, daf-16(mgDF50)X3, daf-2(e1370);daf-16(mu86)X, daf-2(e1370);daf$16(m g D F 47) X 4$ and daf-2(m41);pdk-1(mg142)X. Double mutants were obtained by crossing daf-16(mu86) with daf-18(ok480), akt-1 (ok525), akt-1(mg144) and akt-2(ok393), respectively.

\section{Behavioral integration assay of sensory signals of diacetyl and} $\mathrm{Cu}^{2+}$

To assay the choice between chemotaxis toward diacetyl and the avoidance of $\mathrm{Cu}^{2+}$ in the migration pathway, $20 \mu \mathrm{L} 100 \mathrm{mM} \mathrm{CuSO}_{4}$ solution was spread as a line on the midline of the assay plate $(1 \mathrm{mM}$ $\mathrm{MgSO}_{4}, 1 \mathrm{mM} \mathrm{CaCl}, 5 \mathrm{mM} \mathrm{KPO}_{4}(\mathrm{pH} \mathrm{6.0)}$, and $2 \%$ agar). After $5 \mathrm{~min}, 2 \mu \mathrm{L}$ diluted diacetyl $\left(10^{-2}\right)$ and $1 \mu \mathrm{L} 100 \mathrm{mM} \mathrm{NaN}_{3}$ were spotted on one side of the plate divided by the $\mathrm{Cu}^{2+}$ barrier. $100-300$ young adult worms washed with M9 medium were placed on the other side of the plate. The numbers of animals on the original side $(A)$ and on the odorant side $(B)$ were scored after $90 \mathrm{~min}$ (Ishihara et al., 2002). The choice index was calculated as $A /(A+B) \times 100 \%$. Sensation toward the attractive diacetyl $\left(10^{-2}\right)$ and aversive $\mathrm{Cu}^{2+}$ $(100 \mathrm{mM})$ was assayed in the same way without $\mathrm{Cu}^{2+}$ line and diacetyl spot, respectively.

\section{Body bend and aldicarb resistance assay}

For the body bend assay, worms were tracked for $5 \mathrm{~min}$ at $20^{\circ} \mathrm{C}$ on NGM plates spread with a thin layer of freshly grown OP50 bacteria as described (Cronin et al., 2005). The aldicarb resistance assay was conducted as described by Lackner et al. (Lackner et al., 1999). Worms that failed to respond at all to a harsh somatosensory 
stimulus were classified as paralyzed. Experiments were repeated three times.

\section{Data analysis}

Data analysis was conducted using SigmaStat 3.11 (Systat Software, Inc) and IGOR Pro 5.01 (Wavemetrics, Portland, OR). Results are presented as the mean values \pm SEM., with number of experimental replications $(n)$. Statistical significance was evaluated using the Student's T-test or Mann-Whitney's U-test depending on the normality of the data distribution. Asterisks denote the statistical significance in comparisons with the control: ${ }^{* * *}, p \leqslant 0.001 ;{ }^{* *}, p \leqslant 0.01$; *, $p \leqslant 0.05$.

\section{ACKNOWLEDGEMENTS}

We thank the C. elegans Genetic Center (CGC) for supply of the mutant worms. This work was supported by grants from the National Natural Science Foundation of China (Grant Nos. 30770523, 30630020 and 30670502).

\section{ABBREVIATIONS}

cGMP, cyclic guanosine monophosphate; LDL, low-density lipoprotein; PTEN, phosphatase and tensin homolog deleted on chromosome 10; TGF $\beta$, transforming growth factor beta

\section{REFERENCES}

Bargmann, C.I., Hartwieg, E., and Horvitz, H.R. (1993). Odorantselective genes and neurons mediate olfaction in $C$. elegans. Cell 74, 515-527.

Bargmann, C.I., and Kaplan, J.M. (1998). Signal transduction in the Caenorhabditis elegans nervous system. Annu Rev Neurosci 21, 279-308.

Brenner, S. (1974). The genetics of Caenorhabditis elegans. Genetics 77, 71-94.

Calvert, G.A., Hansen, P.C., Iversen, S.D., and Brammer, M.J. (2001). Detection of audio-visual integration sites in humans by application of electrophysiological criteria to the BOLD effect. Neuroimage 14, 427-438.

Celikel, T., and Sakmann, B. (2007). Sensory integration across space and in time for decision making in the somatosensory system of rodents. Proc Natl Acad Sci U S A 104, 1395-1400.

Colbert, H.A., and Bargmann, C.I. (1995). Odorant-specific adaptation pathways generate olfactory plasticity in $C$. elegans. Neuron 14, 803-812.

Cronin, C.J., Mendel, J.E., Mukhtar, S., Kim, Y.M., Stirbl, R.C., Bruck, J., and Sternberg, P.W. (2005). An automated system for measuring parameters of nematode sinusoidal movement. BMC Genet 6, 5 .
Guo, J., and Guo, A. (2005). Crossmodal interactions between olfactory and visual learning in Drosophila. Science 309, 307-310.

Ikeda, D.D., Duan, Y., Matsuki, M., Kunitomo, H., Hutter, H., Hedgecock, E.M., and lino, Y. (2008). CASY-1, an ortholog of calsyntenins/alcadeins, is essential for learning in Caenorhabditis elegans. Proc Natl Acad Sci U S A 105, 5260-5265.

Ishihara, T., lino, Y., Mohri, A., Mori, I., Gengyo-Ando, K., Mitani, S., and Katsura, I. (2002). HEN-1, a secretory protein with an LDL receptor motif, regulates sensory integration and learning in Caenorhabditis elegans. Cell 109, 639-649.

Kodama, E., Kuhara, A., Mohri-Shiomi, A., Kimura, K.D., Okumura, M., Tomioka, M., lino, Y., and Mori, I. (2006). Insulin-like signaling and the neural circuit for integrative behavior in C. elegans. Genes Dev 20, 2955-2960.

Lackner, M.R., Nurrish, S.J., and Kaplan, J.M. (1999). Facilitation of synaptic transmission by EGL-30 Gqalpha and EGL-8 PLCbeta: DAG binding to UNC-13 is required to stimulate acetylcholine release. Neuron 24, 335-346.

Matsuki, M., Kunitomo, H., and lino, Y. (2006). Goalpha regulates olfactory adaptation by antagonizing Gqalpha-DAG signaling in Caenorhabditis elegans. Proc Natl Acad Sci U S A 103, 1112-1117.

Mohri, A., Kodama, E., Kimura, K.D., Koike, M., Mizuno, T., and Mori, I. (2005). Genetic control of temperature preference in the nematode Caenorhabditis elegans. Genetics 169, 1437-1450.

Paradis, S., and Ruvkun, G. (1998). Caenorhabditis elegans Akt/PKB transduces insulin receptor-like signals from AGE-1 PI3 kinase to the DAF-16 transcription factor. Genes Dev 12, 2488-2498.

Saeki, S., Yamamoto, M., and lino, Y. (2001). Plasticity of chemotaxis revealed by paired presentation of a chemoattractant and starvation in the nematode Caenorhabditis elegans. J Exp Biol 204, 1757-1764.

Solomon, A., Bandhakavi, S., Jabbar, S., Shah, R., Beitel, G.J., and Morimoto, R.I. (2004). Caenorhabditis elegans OSR-1 regulates behavioral and physiological responses to hyperosmotic environments. Genetics 167, 161-170.

Tomioka, M., Adachi, T., Suzuki, H., Kunitomo, H., Schafer, W.R., and lino, Y. (2006). The insulin/PI 3-kinase pathway regulates salt chemotaxis learning in Caenorhabditis elegans. Neuron 51, 613-625.

Ward, S., Thomson, N., White, J.G., and Brenner, S. (2004). Electron microscopical reconstruction of the anterior sensory anatomy of the nematode Caenorhabditis elegans? J Comp Neurol 160, 313-337.

White, F.M., Pereira, L.H., Embil, J.A., and Manuel, F.R. (1986). Ascaris lumbricoides in Nova Scotia. Can J Public Health 77, 201-204.

Zhao, W.Q., Chen, H., Quon, M.J., and Alkon, D.L. (2004). Insulin and the insulin receptor in experimental models of learning and memory. Eur J Pharmacol 490, 71-81. 